\title{
On the Formation of Interstellar Gas Clouds
}

\author{
S. A. KaPLAN
}

Lvov University Observatory, Lvov, U.S.S.R.

$T$ $\mathrm{HE}$ characteristic features of interstellar gas clouds-existence of large density fluctuations, their connection with cosmic dust, stretching along the magnetic fields, and so on-may be described by a shock wave theory in interstellar space.

The author has developed a theory of stationary shock waves accompanied by losses of energy by means of radiation. Choosing two surfaces on both sides of the front, so that the regions of energy radiation should lie between them, we can write an equation for the mass flow and for the impulse conservation between these surfaces, and two equations which determine the stationary temperature of the gas in the field of interstellar radiation. The solution of this system of equations permits one to determine the general changes of thermodynamic and other parameters for the transition of gas through the shock wave with regions of radiative cooling. If changes of the degree of ionization take place, and a magnetic field is present, some terms should necessarily be added to the corresponding equations.

The boundary between the interstellar gas cloud and the intercloud medium must represent the shock wave accompanied by losses of energy by means of radiation, because such ruptures may probably be supposed as the sole explanation of stability of the great density changes (a hundred times and more) often observed in the interstellar space.

In this paper we give some results of the theory of shock waves accompanied by radiative losses of energy.

\section{1.}

The increase of density in such waves is very great, approximately equal to $\rho_{2} / \rho_{1}=v^{2} / R T_{2}$, where $v$ = velocity of the wave, $T_{2}=$ temperature behind the front. For instance, a shock with radiative cooling in an $\mathrm{HI}$ region with a velocity $v$ about $5 \mathrm{~km} / \mathrm{sec}$ at a temperature $T_{2}$ about $100^{\circ}$ causes an increase of density by a factor 30 . This result holds only when the radiative cooling is rather powerful. Such cooling can be maintained in the HI region by the $H_{2}$ molecules forming on particles of cosmic dust. Therefore a sharp increase of density of 30 and more times in the HI region may take place when a sufficient quantity of cosmic dust is available.

\section{2.}

A magnetic field (if it is present) also increases in strength very strongly in a shock wave with radiative cooling. One has $H_{2} / H_{1}=\left(\rho_{2} / \rho_{1}\right) \sin \varphi_{1}$, where $\varphi_{1}=$ the angle the direction of the magnetic field in front of the shock wave and the normal to this front. Both the magnetic field and the direction of the gas flow behind the front are almost parallel to the front of the shock wave considered. Therefore in dense nebulae (and also in filaments) which are formed by the shock waves the flow of gas is mainly directed along the magnetic field.

3.

It is convenient to use the method of self-preserving solutions for the investigation of the motion of the shock wave system. For instance, the problem of propagation of the ionization front [the boundary between (HI) and (HII) regions], considered earlier by $\mathrm{Kahn}^{1}$ and Savedoff and Green ${ }^{2}$ with the aid of other methods, had been solved by the author by means of the method of self-preserving solutions. ${ }^{3}$ The shock wave with radiative cooling which moves in front of the ionization front increases the density in the HI region some hundred times. The ionization wave decreasing the gas density follows behind. The temperature $T_{2}$ and the gas density $\rho_{0}$ behind the ionization front are the determining parameters of this problem. The region of increased density (HI) expands with time and moves with constant velocity. The gas density in this compressed region equals approximately $4 \rho_{0} T_{2} / T_{0}$, where $\rho_{0}$ and $T_{0}$ are the density and temperatures of the gas in the HI region in front of the shock wave. This system of flow is only possible if the condition $\rho_{2}<2 \rho_{0}$ is fulfilled. This is possibly the cause of the instability of this system leading to a disintegration of the dense region into separate clouds, as has been supposed by Oort and Spitzer. ${ }^{4}$

The method of self-preserving solutions was also used for an investigation of the motion in a resisting interstellar medium of envelopes ejected by novae or supernovae.

\section{4.}

In conclusion I should like to make some comments about the connection between the system of cosmic clouds and the interstellar turbulence. According to the author's opinion, magnetogasdynamic turbulence of the different eddies must be more isolated, than in the ordinary turbulence. This hypothesis was suggested to avoid a suppression of motions of the small eddies by the magnetic fields of the big ones. Such suppression contradicts the modern statistical picture of turbulence.

If this hypothesis is correct, it is possible to interpret the interstellar gas clouds as more or less isolated eddies of interstellar magnetic turbulence.

\footnotetext{
${ }^{1}$ F. D. Kahn, Bull. Astron. Soc. Neth. 12, 456 (1954).

2 M. Savedoff and J. Green, Astrophys. J. 122, 477 (1955)

3 S. A. Kaplan, 1952, Doklady Akad. Nauk S.S.S.R. 87, 909 (1952); Astron. Zhur. 31, 31 (1954); Zhur. Exptl. i Theoret. Fiz. 27, 699 (1954)

${ }^{4}$ J. H. Oort and L. Spitzer, Astrophys. J. 121, 6 (1954).
} 